\title{
A Transient Analysis of Negative Refraction at the Interface Between Two Transmission-Line Grids
}

\author{
Loïc Markley and George V. Eleftheriades \\ The Edward S. Rogers Sr. Department of Electrical and Computer Engineering \\ University of Toronto, 10 King's College Road \\ Toronto, Ontario, M5S 3G4, Canada
}

\begin{abstract}
In response to a few recent publications that deny the existence of negative refraction at a single interface on the grounds that it violates causality, an investigation is performed using two-dimensional transmission-line grids. Containing only passive elements and simulated using a standard circuit simulator, the transient response of an obliquely incident Gaussian beam on a negative-refractive-index material is examined.

Phase velocity and group velocity (power) are both shown to refract negatively. The refracted beam front, however, is no longer parallel to the phase fronts. In order to remain causal, it must always maintain a positive angle with respect to the interface. This front is called the group front, and despite facing in a different direction, it propagates in the direction of the group velocity.

The effect of adding a second interface to the simulation is also shown. In this case, the wave emerging from the negativerefractive-index slab is parallel to the incident wave.

Index Terms - negative refraction, transmission lines, metamaterials, group fronts, group velocity, dispersion
\end{abstract}

\section{INTRODUCTION}

The study of materials with negative indices of refraction was carried out systematically in 1967 when Victor Veselago published his paper on materials with simultaneously negative permittivity and permeability [1]. He observed that under these conditions, Maxwell's equations are satisfied only if the negative square root is taken when calculating the index of refraction $n=-\sqrt{\epsilon \mu}$. This implied from the electromagnetic boundary conditions that a wave obliquely incident from freespace would refract negatively as it passed into such a material. At the time of publication, negative-refractive index materials were purely hypothetical so it wasn't until Sir John Pendry published his paper on the "perfect lens" in 2000 [2] that the phenomenon of negative refraction began to be more carefully scrutinized.

Although over ten years have passed since the first experimental verification of negative refraction [3], the very reality of it is still being challenged by members of the microwave community. Several works in the recent literature have even claimed that negative refraction is a physical impossibility [4], [5], despite their arguments having been raised and addressed by the community many years ago [6], [7]. The purpose of this paper is to revisit and clarify a few aspects of negative refraction at a single interface related to causality that have caused confusion and misunderstandings. This work studies the transient evolution of a negatively refracted wavefront

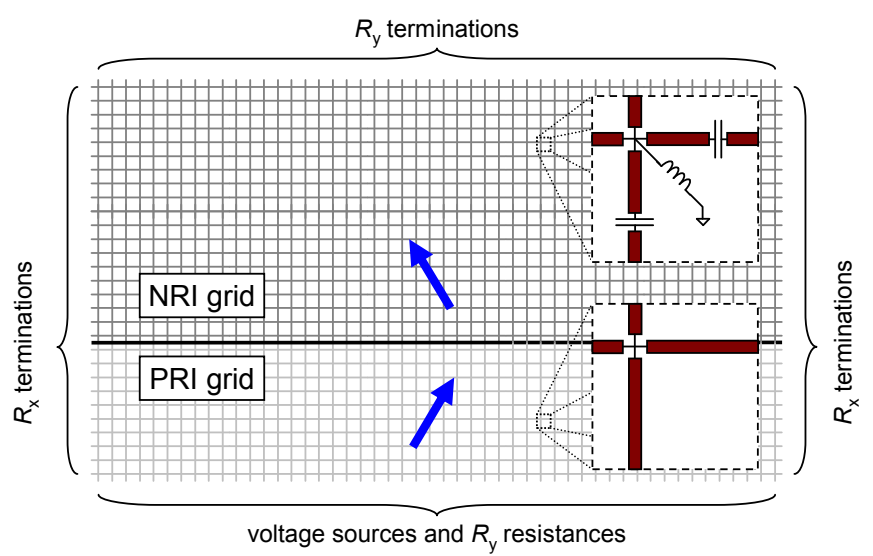

Fig. 1. (color online) A diagram of the transmission-line grid simulation domain along with a close up of each unit cell.

propagating along a two-dimensional negative-refractive-index transmission-line grid. Although this is not the first transient investigation of negative refraction [8], it is the first that is not based on idealized Drude permittivity and permeability models, and the first carried out in transmission-line metamaterials. The transient response is investigated using a circuit grid made from inductors, capacitors, and simple transmission-line segments only. Resistors are added to terminate the grid, and voltage sources are used along one edge to excite a voltage wave. The circuit is solved using the standard circuit simulator found within Agilent's Advanced Design System. The incident and refracted voltage waves are then directly observed.

\section{TRANSMISSION-LINE GRID DESIGN}

Figure 1 shows a diagram of the simulation domain used to investigate negative refraction. A two-dimensional positiverefractive-index (PRI) transmission-line grid is connected to a negative-refractive-index (NRI) transmission-line grid with the grids along each domain edge terminated by the Bloch resistance. A wave is excited into the PRI medium from voltage sources placed along the bottom edge of the domain. The node voltages at each unit cell are then recorded to monitor the wave propagation and refractive behavior at the interface. The NRI grid is designed by periodically loading a host transmission-line grid with series capacitors and shunt 


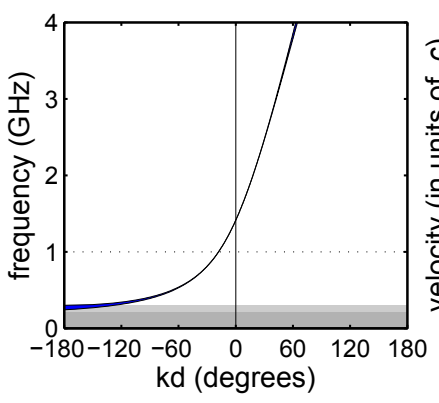

(a)

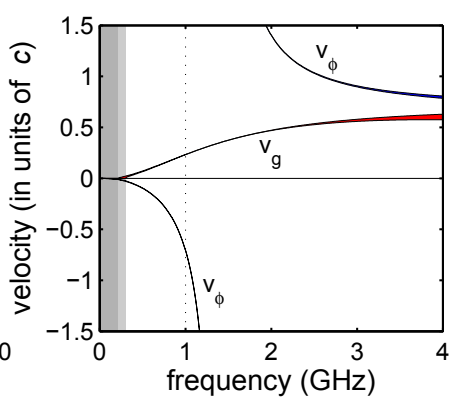

(b)
Fig. 2. (color online) The dispersion diagram (a) and phase and group velocities (b) are plotted for wave propagation in the NRI transmission-line medium. The anisotropy is indicated by the spreading in the curves while the shaded regions represent the stop bands.

TABLE I

PhASE AND GROUP VELOCITIES AT $1 \mathrm{GHz}$

\begin{tabular}{|c|c|c|}
\hline PRI region & $v_{\phi, 1}=0.7056 c=u$ & $v_{\mathrm{g}, 1}=0.7027 c=0.9958 u$ \\
\hline NRI region & $v_{\phi, 2}=-0.7056 c=-u$ & $v_{\mathrm{g}, 2}=0.2317 c=0.3283 u$ \\
\hline
\end{tabular}

inductors [9], [10] while the PRI grid is simply the unloaded host transmission-line grid.

The grids were designed to support a propagating wave with a characteristic impedance of $50 \Omega$ and a per-unit-cell phase shift of $|k d|=18^{\circ}$ for on-axis propagation at $1 \mathrm{GHz}$. This corresponded to a unit cell size of $\lambda / 20$. The host transmissionline segments had a per-unit-cell phase shift of $\beta d=12.702^{\circ}$ and a characteristic impedance of $71.153 \Omega$, with the NRI grid loaded by $5.0243 \mathrm{pF}$ capacitors and $12.718 \mathrm{nH}$ inductors.

The dispersion diagram for the NRI transmission-line grid is plotted in Fig. 2(a) with the corresponding phase and group velocities plotted in Fig. 2(b). Lower frequency components travel slower than higher ones, with no stop band between the backward and forward wave regions. The phase velocity $v_{\phi}=$ $\omega / k$ and group velocity $v_{\mathrm{g}}=\partial \omega / \partial k$ at the design frequency are listed in Table I, where $u$ is the magnitude of the phase velocity at the design frequency and $c=\omega / \beta$ is the phase velocity along the host transmission-line segments. Although the grids are not isotropic, when the unit cell dimension is much smaller than the wavelength (i.e. near the $k d=0$ point), the anisotropy is negligible. In particular, at $1 \mathrm{GHz}, v_{\phi}$ and $v_{\mathrm{g}}$ are constant with respect to the direction of propagation to within $0.2 \%$ and $0.6 \%$, respectively.

\section{REFRACTION INTO AN NRI MEDIUM}

An obliquely incident wave is shown refracting into a dispersive medium in Fig. 3(a). Since the medium is effectively isotropic, the propagation vector is parallel to both the phase velocity and the group velocity. The phase fronts are perpendicular to the phase velocity, but we can see that the group fronts are only perpendicular to the group velocity when there is no dispersion. By connecting points of simultaneity as they move with the group velocity, the energy in the

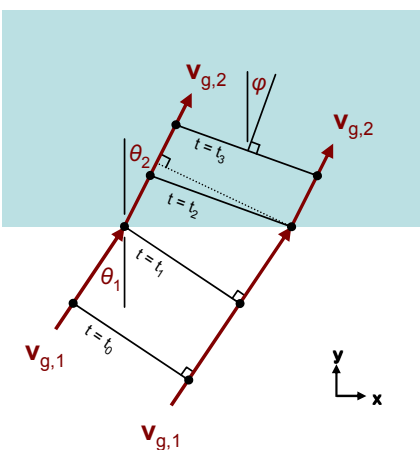

(a)

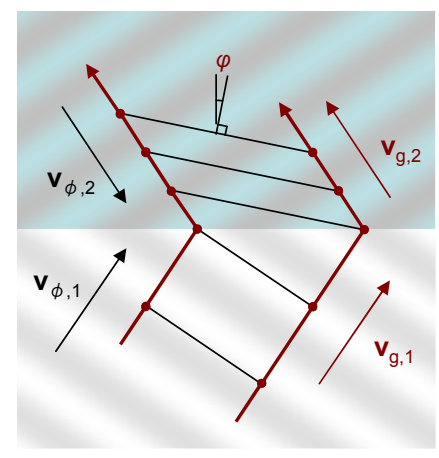

(b)
Fig. 3. (color online) Diagrams illustrating the refraction of group fronts into (a) a general isotropic dispersive medium and (b) an isotropic NRI medium. The group fronts move with the group velocity $\mathbf{v}_{\mathrm{g}}$ but make an angle $\phi$ with the interface (where $\phi \neq \theta_{2}$ ). The background shading in (b) shows the phase fronts.

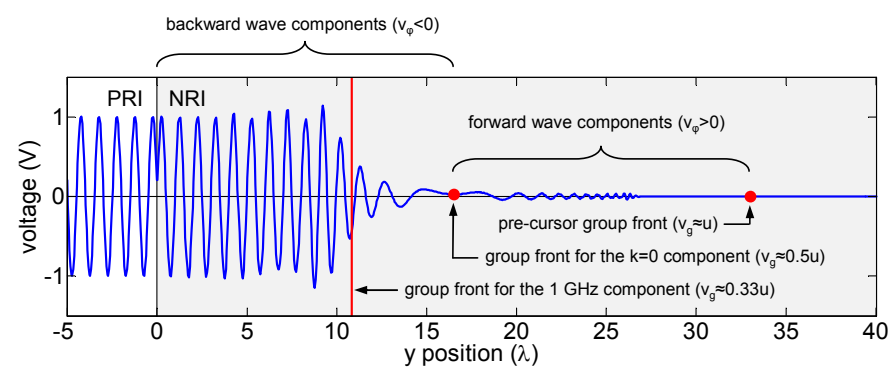

Fig. 4. (color online) Simulation results for a plane wave at normal incidence with various group fronts indicated.

wave can be tracked from $t_{0}$ to $t_{3}$. As the group velocity changes going into the dispersive medium, these group fronts must change angle, but to ensure causality, they must always maintain a positive angle with respect to the interface. In other papers that examine the interference between two negatively refracting waves, the group fronts have been characterized by an interference velocity [11] or a group front velocity [12], but these terms are misleading since the group fronts don't actually propagate in the direction of these velocities. They propagate, rather, in the direction of the group velocity and therefore need only to be characterized by the angle $\phi$ they take with the interface. This angle was derived geometrically from Fig. 3(a) and expressed in (1), where $\phi-\theta_{2}$ describes the tilt between the group fronts and the phase fronts. The direction can also be calculated by taking the partial derivative $\partial \mathbf{k}_{2} / \partial \omega$, where $\mathbf{k}_{2}$ is the refracted propagation vector given a fixed incidence angle $\theta_{1}$. It must be emphasized that this refractive behavior is not unique to NRI media, but is a general consequence of refraction into a dispersive medium [13].

$$
\tan \left(\phi-\theta_{2}\right)=\frac{\sin \theta_{1}}{\cos \theta_{2}}\left(\frac{v_{\mathrm{g}, 2}}{v_{\mathrm{g}, 1}}-\frac{v_{\phi, 2}}{v_{\phi, 1}}\right)
$$

\section{TRANSIENT SIMULATION RESULTS}

Figure 4 plots the voltage along $y$ for a $1 \mathrm{GHz}$ plane wave at normal incidence $38.1 \mathrm{~ns}$ after it is turned on. Three group 
fronts have been indicated on the figure, with the zero-index group front dividing the faster forward-wave components from the slower backward-wave components. The pre-cursors travel the quickest with a speed of around $u$ while the $1 \mathrm{GHz}$ group front travels at around $0.33 u$ and marks the front of the plane wave.

In order to simulate negative refraction, a $4 \lambda \times 4 \lambda$ simulation domain was used with the PRI-NRI interface one wavelength from the bottom of the domain $(81 \times 21$ PRI unit cells and $81 \times 61$ NRI unit cells). The edges of the domain were Bloch terminated with $R_{\mathrm{x}}=194.8 \Omega$ and $R_{\mathrm{y}}=51.8 \Omega$ resistors, while the voltage sources excited a $1 \mathrm{GHz}$ Gaussian beam with a $1.5 \lambda$ waist at an incidence angle of $15^{\circ}$. The Gaussian beam was turned on at $t=0 \mathrm{~ns}$ in such a way as to produce a beam front parallel to the phase front in the PRI region.

Negative refraction is shown in Fig. 5 at various time steps to show the evolution of the beam in the NRI medium. The group front for the $1 \mathrm{GHz}$ component was calculated using (1) to have $\phi=4.61^{\circ}$ and is highlighted in white along with the pre-cursor group front. At $1.55 \mathrm{~ns}$, the beam begins to cross into the NRI medium starting from the left side. At $2.77 \mathrm{~ns}$, negative phase fronts can be seen below the $1 \mathrm{GHz}$ group front while close to the pre-cursor group front several positively refracting phase fronts are just visible. These fronts correspond to quickly moving high-frequency components which barely slow as they pass through the interface. At $5.76 \mathrm{~ns}$ and $8.74 \mathrm{~ns}$, the beam continues to refract negatively while the group front maintains a positive angle with respect to the interface.

\section{REFRACTION THROUGH A SLAB OF NRI MATERIAL}

It is also very instructive to examine the behavior of a wave refracting through a negative-refractive-index region of finite extent. In this case an NRI slab is placed between two PRI regions with a wave incident at an oblique angle, as illustrated in Fig. 6. As in the single interface scenario, the phase velocity and group velocity remain parallel throughout the domain. The phase fronts are perpendicular to the phase velocity while the group fronts are only perpendicular to the group velocity when the region is non-dispersive. The group fronts move with the group velocity from $t_{0}$ to $t_{7}$, changing orientation as they move into the NRI medium but always maintaining a positive angle with respect to the interfaces. As the wave exits the slab at the second interface, the fronts return to their original orientation, with the phase and group velocities parallel to those of the incident wave.

The two-interface circuit simulations were setup using the same PRI and NRI unit cells as for the single interface simulations. The wave excitation and edge terminations also remained the same. The simulation domain still measured $4 \lambda \times 4 \lambda$ but now a second interface was introduced at a distance of $1.5 \lambda$ away from the first. The domain was therefore separated into a lower PRI region of $81 \times 21$ unit cells, a middle NRI region of $81 \times 30$ unit cells, and a upper PRI region of $81 \times 31$ unit cells. Four snapshots were taken as the wave propagated through the domain. The first two were taken at $1.55 \mathrm{~ns}$ and $2.77 \mathrm{~ns}$, but since the wave had only passed through the first interface, the fields were the same as the first two snapshots in Fig. 5. The last two snapshots are shown in Fig. 7. The thin and thick white lines indicate the position of the pre-cursor group front and the design frequency group front, respectively. The pre-cursors are almost completely unaffected by the presence of the NRI material, passing through the slab without slowing or refracting and exiting in line with the incident beam (see the fields at $4.55 \mathrm{~ns}$ ). The $1 \mathrm{GHz}$ group front travels much slower through the slab, maintaining causality with a positive angle $\phi$ and emerging on the other side of the slab parallel to the original group fronts. As expected from refraction, the angle of emergence from the slab is equal to the angle of incidence. This is in contrast to the construct presented in [5] where these angles have opposite signs, suggesting phase conjugation rather than refraction.
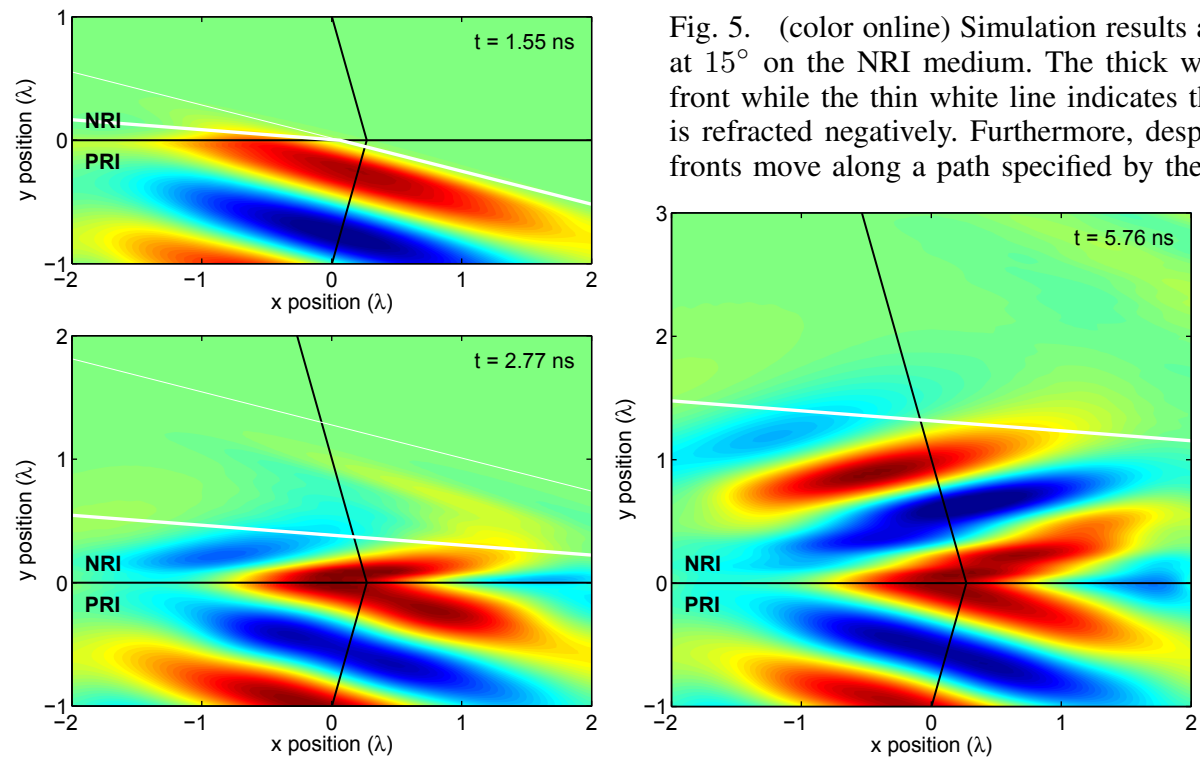

Fig. 5. (color online) Simulation results at four time steps for a Gaussian beam incident at $15^{\circ}$ on the NRI medium. The thick white line indicates the design-frequency group front while the thin white line indicates the pre-cursor group front. Note that the power is refracted negatively. Furthermore, despite pointing in a different direction, the group fronts move along a path specified by the group velocity vector $\mathbf{v}_{\mathrm{g}}$.

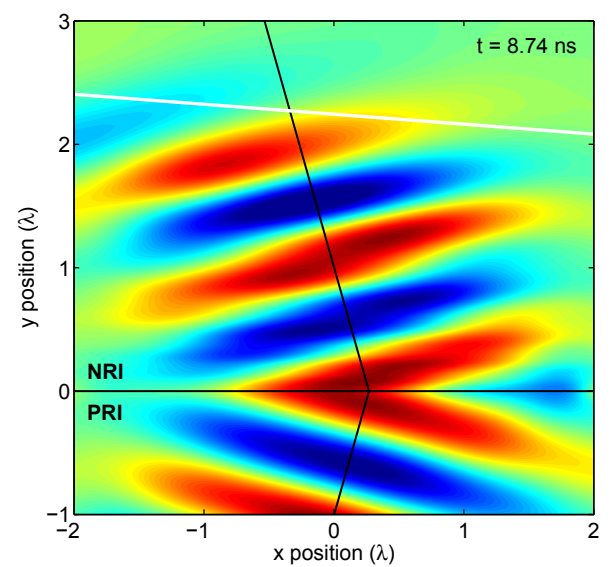


Fig. 6. (color online) An illustration of the properties of a wave refracting through an isotropic NRI slab. After the second interface, the wave is parallel to the incident wave and the group fronts are once again perpendicular to the group velocity.

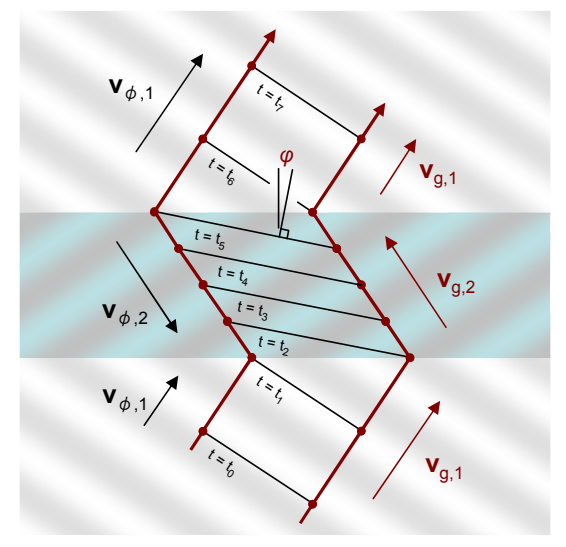

Fig. 7. (color online) The simulated voltage wave at two time steps for a Gaussian beam incident at $15^{\circ}$ on an NRI slab. At the earlier time steps of $1.55 \mathrm{~ns}$ and $2.77 \mathrm{~ns}$, the wave has only refracted through the first interface and the fields are the same as in Fig. 5. The thick white line indicates the design-frequency group front while the thin white line indicates the pre-cursor group front. Note that although the power is refracted negatively, the group fronts make a positive angle with the interfaces. Furthermore, when the beam emerges on the other side of the slab, it propagates parallel to the incident wave.
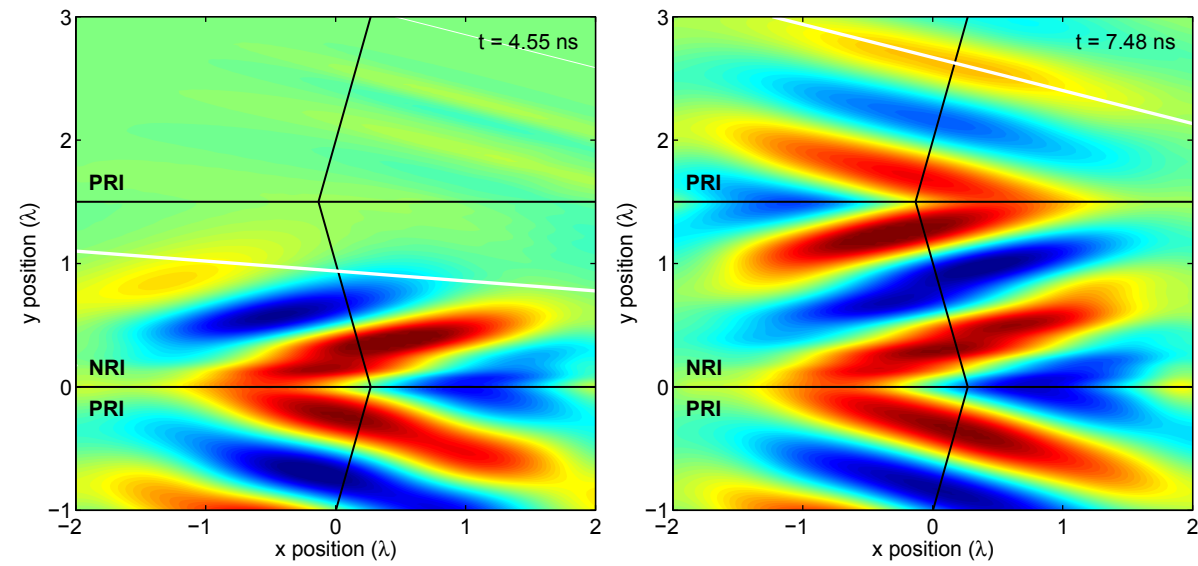

\section{CONCLUSION}

Negative refraction at the interface between two transmission-line grids has been investigated in the time domain. Through a careful analysis of dispersive refraction, it was shown that the group fronts are not perpendicular to the group velocity, and in fact must always maintain a positive angle to the interface to satisfy causality. This in no way, however, restricts the phase and group velocities from refracting negatively. Circuit simulations demonstrating negative refraction were shown at various time steps to demonstrate the causal evolution of the refracted wave. A simulation showing a wave refracting through a negativerefractive-index slab was also presented. This paper was written in response to publications that claim negative refraction is impossible. Hopefully, these results can clear up some of the confusion surrounding negative refraction and eliminate any remaining doubts in the microwave community. The root of this confusion seems to stem from the fact that in these reports (e.g. [4], [5]) dispersion is ignored and causality is examined with respect to the phase fronts rather than the group fronts.

\section{REFERENCES}

[1] V. G. Veselago, "The electrodynamics of substances with simultaneously negative values of $\epsilon$ and $\mu$," Sov. Phys. Usp., vol. 10, no. 4, pp. 509-514, Jan./Feb. 1968.

[2] J. B. Pendry, "Negative refraction makes a perfect lens," Phys. Rev. Lett., vol. 85 , no. 18 , pp. 3966-3969, Oct. 2000.

[3] R. A. Shelby, D. R. Smith, and S. Schultz, "Experimental verification of a negative index of refraction," Science, vol. 282, no. 4, pp. 77-79, Apr. 2001.

[4] B. A. Munk, Metamaterials: critique and alternatives. Hoboken, New Jersey: John Wiley \& Sons, Inc., 2009.

[5] I. C. Hunter, A. I. Abunjaileh, J. D. Rhodes, R. V. Snyder, and M. Meng, "Propagation and negative refraction," IEEE Microwave Magazine, vol. 13, no. 5, pp. 58-65, 2012.
[6] P. M. Valanju, R. M. Walser, and A. P. Valanju, "Wave refraction in negative-index media: always positive and very inhomogeneous," Phys. Rev. Lett., vol. 88, no. 18, p. 187401, May 2002.

[7] J. B. Pendry and D. R. Smith, "Comment on "Wave refraction in negative-index media: always positive and very inhomogeneous"," Phys. Rev. Lett., vol. 90, no. 2, p. 029703, Jan. 2003.

[8] J. Pniewski and T. Szoplik, "Group front evolution of Gaussian beam refracted from a right- to left-handed medium," Opt. Express, vol. 14, no. 18, pp. 8232-8239, Sept. 2006.

[9] G. V. Eleftheriades, A. K. Iyer, and P. C. Kremer, "Planar negative refractive index media using periodically $L-C$ loaded transmission lines," IEEE Trans. Microwave Theory Tech., vol. 50, no. 12, pp. 2702 2712, Dec. 2002.

[10] A. Grbic and G. V. Eleftheriades, "Periodic analysis of a 2-D negative refractive index transmission line structure," IEEE Trans. Antennas Propagat., vol. 51, no. 10, pp. 2604-2611, Oct. 2003.

[11] D. R. Smith, D. Schurig, and J. B. Pendry, "Negative refraction of modulated electromagnetic waves," Appl. Phys. Lett., vol. 81, no. 15, pp. 2713-2715, Oct. 2002.

[12] Z. M. Zhang and K. Park, "On the group front and group velocity in a dispersive medium upon refraction from a non-dispersive medium," Journal of Heat Transfer, vol. 126, pp. 244-249, Apr. 2004.

[13] J. Hebling, "Derivation of the pulse front tilt caused by angular dispersion," Opt. Quant. Electron., vol. 28, pp. 1759-1763, July 1996. 